\title{
The effects of vitamin D supplementation on metabolic profiles and gene expression of insulin and lipid metabolism in infertile polycystic ovary syndrome candidates for in vitro fertilization
}

Majid Dastorani ${ }^{1}$, Esmat Aghadavod ${ }^{1 *}$, Naghmeh Mirhosseini ${ }^{2}$, Fatemeh Foroozanfard ${ }^{3}$, Shahrzad Zadeh Modarres ${ }^{4}$, Mehrnush Amiri Siavashani ${ }^{5}$ and Zatollah Asemi ${ }^{{ }^{*}}$

\begin{abstract}
Background: Vitamin D deficiency in women diagnosed with polycystic ovary syndrome (PCOS) remarkably decreases the chance of pregnancy, which might be related to its impact on metabolic abnormalities in these patients. It is hypothesized that vitamin D supplementation influences metabolic profile of these patients and indirectly might affect fertility and the outcomes. Therefore, this study was conducted to determine the effects of vitamin D supplementation on the levels of anti-Müllerian hormone (AMH), metabolic profiles, and gene expression of insulin and lipid metabolism in infertile women with PCOS who were candidate for in vitro fertilization (IVF).
\end{abstract}

Methods: This study was a randomized, double-blinded, placebo-controlled trial conducted among 40 infertile women, aged 18-40 years, diagnosed with PCOS and was candidate for IVF. Participants were randomly assigned into two intervention groups for receiving either $50,000 \mathrm{IU}$ vitamin D or placebo ( $n=20$ each group) every other week for 8 weeks. Gene expression for insulin and lipid metabolism was conducted using peripheral blood mononuclear cells (PBMCs) of women with PCOS, via RT-PCR method.

Results: Vitamin D supplementation led to a significant reduction in serum $\mathrm{AMH}(-0.7 \pm 1.2 \mathrm{vs} .-0.1 \pm 0.5 \mathrm{ng} / \mathrm{mL}$, $P=0.02$ ), insulin levels $(-1.4 \pm 1.6$ vs. $-0.3 \pm 0.9 \mu \mathrm{lU} / \mathrm{mL}, P=0.007)$, homeostatic model of assessment for insulin resistance $(-0.3 \pm 0.3$ vs. $-0.1 \pm 0.2, P=0.008)$, and a significant increase in quantitative insulin sensitivity check index $(+0.009 \pm 0.01$ vs. $+0.001 \pm 0.004, P=0.04)$, compared with the placebo. Moreover, following vitamin $D$ supplementation there was a significant decrease in serum total- $(-5.1 \pm 12.6 \mathrm{vs} .+2.9 \pm 10.9 \mathrm{mg} / \mathrm{dL}, P=0.03)$ and $\mathrm{LDL}$-cholesterol levels $(-4.5 \pm 10.3 \mathrm{vs}$. $+2.5 \pm 10.6 \mathrm{mg} / \mathrm{dL}, \mathrm{P}=0.04)$ compared with the placebo.

Conclusion: Overall, the findings of this trial supported that 50,000 IU vitamin D supplementation every other week for 8 weeks had beneficial effects on insulin metabolism, and lipid profile of infertile women with PCOS who are candidate for IVF. These benefits might not be evident upon having sufficient vitamin D levels.

Trial registration: This study was retrospectively registered in the Iranian website (www.irct.ir) for clinical trials registration (http://www.irct.ir: IRCT20170513033941N27).

Keywords: Vitamin D supplementation, Glycemic control, Cardio-metabolic, In vitro fertilization

\footnotetext{
*Correspondence: aghadavod_m@yahoo.com; asemi_r@yahoo.com; asemi_z@Kaums.ac.ir

${ }^{1}$ Research Center for Biochemistry and Nutrition in Metabolic Diseases,

Kashan University of Medical Sciences, Kashan, I.R, Iran

Full list of author information is available at the end of the article
}

(C) The Author(s). 2018 Open Access This article is distributed under the terms of the Creative Commons Attribution 4.0 International License (http://creativecommons.org/licenses/by/4.0/), which permits unrestricted use, distribution, and

reproduction in any medium, provided you give appropriate credit to the original author(s) and the source, provide a link to the Creative Commons license, and indicate if changes were made. The Creative Commons Public Domain Dedication waiver (http://creativecommons.org/publicdomain/zero/1.0/) applies to the data made available in this article, unless otherwise stated. 


\section{Background}

Polycystic ovary syndrome (PCOS) is one of the most common endocrine disorders among women affecting $4 \%$ to $12 \%$ of women in reproductive age [1]. It is a multifactorial syndrome presented with obesity, insulin resistance, dyslipidemia and other metabolic abnormalities. Obesity leads to more than $70 \%$ insulin resistance in these patients [2]. Insulin resistance, occurs in patients with PCOS, is significantly associated with different metabolic abnormalities including elevated aromatase activity, increased androgen production, and impaired progesterone synthesis in granulosa cells [2]. Current evidence has reported a high risk of metabolic disorders predisposing individuals to cardiovascular disease (CVD) in in vitro fertilization (IVF) pregnancies [3, 4] and animal models $[5,6]$. However, the independent role of insulin resistance in in vitro fertilization (IVF) outcomes is less defined in the literature [7].

There is a significant association between serum vitamin D levels and reproductive function, already reported in murine models [8]. Further, studies investigating the role of vitamin $\mathrm{D}$ receptor (VDR) in reproductive tissues have supported a positive correlation between vitamin $\mathrm{D}$ status and reproduction [9]. VDR is located in different reproductive tissues including ovary (particularly granulosa cells), uterus, placenta and testis [10]. Diverse expression of VDR suggests a potential role of vitamin D in female reproductive function [11]. Moreover, in PCOS, ovarian physiology is influenced by the metabolic disorders observed in these patients; therefore, a beneficial effect of vitamin $\mathrm{D}$ on metabolic abnormalities might translate into an ideal ovarian physiology $[12,13]$. Vitamin D supplementation is recommended as a potential therapeutic adjunct for the ovulatory dysfunction and metabolic disorders observed in women with PCOS $[14,15]$. Evidence evaluating the impact of vitamin D deficiency on the success rate of reproduction following IVF in humans is limited [16, 17]. Moreover, vitamin D deficiency is correlated with poor ovarian stimulation in PCOS but not unexplained infertility [18]. Likewise, vitamin D has been shown to be involved in a minor pathway related to metabolic and hormonal dysregulation in women with PCOS [19]. Therefore, the beneficial effects of vitamin $\mathrm{D}$ on the metabolic disorders in PCOS might translate into an improvement in ovarian physiology. The majority of current studies investigating the effects of vitamin D supplementation on metabolic status in patients with PCOS have not specifically looked into IVF. And to our best knowledge, this trial is the first study assessing the effects of vitamin D supplementation on glycemic control, markers of cardiometabolic risk and gene expression of insulin and lipid metabolism in infertile women diagnosed with PCOS who were candidate for IVF. In a study conducted by Seyyed Abootorabi et al. [20] vitamin D supplementation at a dosage of
50,000 IU/week for 8 weeks reduced fasting glucose and increased adiponectin levels in women diagnosed with PCOS who were vitamin D deficient. Further, in a meta-analysis performed by Xue et al. [21], vitamin D administration to patients with PCOS significantly decreased triglycerides levels, while did not change insulin metabolism and other markers of lipid profiles. On the other hand, recently there are concerns about the risks associated to uncontrolled vitamin D supplementation [22].

Current evidence and the physiological role of vitamin $\mathrm{D}$ in reproductive activity might support the significance of vitamin D supplementation while doing IVF in infertile women with PCOS F. So, this study was aimed to determine the effects of vitamin $\mathrm{D}$ supplementation on glycemic control, markers of cardiometabolic abnormalities and gene expression of insulin and lipid metabolism in infertile women diagnosed with PCOS and candidate for IVF.

\section{Methods}

Trial design and participants' characteristics

This study was a randomized, double-blinded, placebocontrolled trial conducted on 40 infertile women, aged 18 to 40 years old, with PCOS, diagnosed using Rotterdam criteria [23], who were candidate for IVF. The study was registered in the Iranian website for clinical trials registration (http://www.irct.ir: IRCT20170513033941N2 7). Recruited participants were referred patients at Research and Clinical Center for Infertility and Naghavi Clinic, Kashan, Iran, from December 2017 through March 2018. Any case of metabolic disorders, including thyroid disorder, diabetes or impaired glucose tolerance was excluded at screening phase. This study was performed following Declaration of Helsinki and was approved by the research ethics committee of Kashan University of Medical Sciences (KAUMS). Written informed consent was signed by all subjects prior to the intervention.

\section{Intervention}

Participants were randomly assigned into two intervention groups to receive either 50,000 IU vitamin D $(n=$ $20)$ or placebo $(n=20)$ every other week for 8 weeks. Vitamin D and placebo (paraffin) capsules were similarly matched in color, shape, size and packaging, and were manufactured by Zahravi (Tabriz, Iran) and Barij Essence (Kashan, Iran), respectively. Random number table was used for randomization by one of the researchers not involved in other processes of trial. Randomization and allocation were concealed from the investigators and study participants until the completion of analyses. Compliance rate was assessed through measuring serum $25(\mathrm{OH}) \mathrm{D}$ levels and asking patients 
to return back the medication containers. To increase the compliance, all subjects were received a short message on their cell phones every day, throughout the trial, to take the supplements.

\section{Assessment of outcomes}

Insulin metabolism was considered as the primary outcome. Lipid profiles and gene expression of insulin and lipid metabolism were defined as the secondary outcomes. Fifteen milliliter fasting blood was collected at the beginning and end of the trial at Naghavi Clinic laboratory, Kashan, Iran. Fasting plasma glucose (FPG) concentrations were measured on the day of blood collection, using enzymatic kits with the coefficient variances (CVs) of less than 5\%. Serum 25-hydroxyvitamin D $(25(\mathrm{OH}) \mathrm{D})$ concentrations were determined by an ELISA kit (IDS, Boldon, UK) with inter- and intra-assay CVs of lower than 7\%. Serum AMH levels were measured using an ELISA kit (Bioactiva, Homburg, Germany) with CVs of lower than 6\%. Serum insulin concentrations were measured by an ELISA kit (DiaMetra, Milano, Italy) with inter- and intra-assay CVs of 3.5 and 5.0\%, respectively. The homeostasis model of assessment-insulin resistance (HOMA-IR) and the quantitative insulin sensitivity check index (QUICKI) were determined according to the published formula [24]. Enzymatic kits (Pars Azmun, Tehran, Iran) were applied to measure serum lipid profiles with inter- and intra-assay of less than $5 \%$.

\section{Isolation of lymphocyte cells}

Blood samples were used to extract lymphocyte cells, using 50\% percoll (Sigma-Aldrich, Dorset, UK). Cell count and viability test were performed using trypan blue, RNA and DNA extraction [25].

\section{RNA extraction and real-time PCR (RT-PCR)}

RNA was extracted from blood samples using RNX-plus kit (Cinnacolon, Tehran, Iran). RNA suspension was frozen at $-20{ }^{\circ} \mathrm{C}$ until cDNA was derived. RNA quantification was conducted using UV spectrophotometer and total RNAs were extracted from each sample. Each sample OD 260/280 ratio was considered to range from 1.7 to 2.1 , representing no contamination with either protein or DNA [25].

The isolated RNA was reverse transcribed to cDNA library, using moloney murine leukemia virus reverse transcriptase (RT). Gene expressions of peroxisome proliferator-activated receptor gamma (PPAR- $\gamma$ ), glucose transporter 1 (GLUT-1) and low-density lipoprotein receptor (LDLR) were done using peripheral blood mononuclear cells (PBMCs) via SYBR green detection and Amplicon Kit, applying quantitative RT-PCR and Light Cycler technology (Roche Diagnostics, Rotkreuz, Switzerland) (Table 1). Glyceraldehyde-3-phosphate dehydrogenase (GAPDH) primers were used as a housekeeping gene. Primers were designed using Primer Express Software (Applied Biosystems, Foster City, USA) and Beacon designer software (Takaposizt, Tehran, Iran). Relative transcription levels were calculated using Pffafi or $2^{-\Delta \Delta C T}$ methods [25].

\section{Sample size}

Clinical trial sample size formula was used to calculate sample size considering a type one error $(\alpha)$ of 0.05 and type two error ( $\beta$ ) of 0.20 with the power of $80 \%$. Mean difference (d) of HOMA-IR equal to 0.8 and SD of 0.8 were used for the calculation [26]. Sample size was defined as 16 participants in each group. Considering 4 probable dropouts in each group, the final sample size was determined to be 20 participants in each group.

\section{Statistical methods}

Kolmogorov-Smirnov test was used to assess the normal distribution of variables. Analyses were replicated using intention-to-treat (ITT) approach. To determine the differences in anthropometric measures and gene expression of insulin and lipid metabolism between the intervention groups, we used independent samples $t$-test. The effects of vitamin D supplementation on glycemic control and markers of cardiometabolic risk were assessed using repeated measures ANOVA test. The $P$-values of $<0.05$ were considered statistically significant. Statistical analyses were conducted using the Statistical Package for Social Science version 18 (SPSS Inc., Chicago, Illinois, USA).

\section{Results}

We had three dropouts in each intervention group due to personal reasons, and 34 participants [infertile women with PCOS candidate for IVF receiving vitamin D $(n=$ 17) and placebo $(\mathrm{n}=17)$ ] completed the study (Fig. 1). Using ITT protocol, all 40 participants (20 in each group) were included in the final analysis. Compliance rate in this trial ranged $90 \%$ to $100 \%$ in both groups. No side effects were reported following vitamin D supplementation in infertile women with PCOS who were candidate for IVF throughout the study. Mean age, height, weight and BMI were not statistically different between the intervention groups throughout the trial (Table 2).

After 8-week intervention, vitamin D supplementation led to a significant reduction in serum AMH $(-0.7 \pm 1.2$ vs. $-0.1 \pm 0.5 \mathrm{ng} / \mathrm{mL}, P=0.02)$ and insulin levels $(-1.4 \pm$ 1.6 vs. $-0.3 \pm 0.9 \mu \mathrm{IU} / \mathrm{mL}, P=0.007)$, HOMA-IR $(-0.3 \pm$ 0.3 vs. $-0.1 \pm 0.2, P=0.008)$, as well as a significant increase in QUICKI $(+0.009 \pm 0.01$ vs. $+0.001 \pm 0.004, P=$ 0.04) compared with placebo (Table 3). Further, taking vitamin $\mathrm{D}$ supplements significantly decreased serum total- $(-5.1 \pm 12.6$ vs. $+2.9 \pm 10.9 \mathrm{mg} / \mathrm{dL}, P=0.03)$ and 
Table 1 Specific primers used for real-time quantitative PCR

\begin{tabular}{llcr}
\hline Gene & Primer & Product size $(\mathrm{bp})$ & Annealing temperature $\odot$ \\
\hline GAPDH & F: AAGCTCATTTCCTGGTATGACAACG & 126 & 61.3 \\
& R: TCTTCCTCTTGTGCTCTTGCTGG & & 54 \\
PPAR-y & F: ATGACAGACCTCAGACAGATTG & 210 & \\
& R: AATGTTGCAGTGGCTCAG & 238 \\
GLUT-1 & F: TATCTGAGCATCGTGGCCAT & & 62.1 \\
LDLR & F: ACTTACACGTAGGGACCACACAG & 223 & 57 \\
& R: GGCCACACATCCCATGATTC & &
\end{tabular}

$\overline{G A P D H}$ glyceraldehyde-3-Phosphate dehydrogenase, GLUT-1 glucose transporter 1, LDLR low-density lipoprotein receptor, PPAR- $y$ peroxisome proliferator-activated receptor gamma

LDL-cholesterol levels $(-4.5 \pm 10.3$ vs. $+2.5 \pm 10.6 \mathrm{mg} /$ $\mathrm{dL}, \mathrm{P}=0.04)$ compared with the placebo. There was no significant effect of vitamin D supplementation on fasting glucose and other parameters of lipid profiles.

RT-PCR quantitative tests showed a significant upregulation of gene expression of PPAR- $\gamma(P=0.01)$, GLUT$1(P=0.009)$ and LDLR $(P=0.03)$ in PBMCs of infertile women diagnosed with PCOS who were candidate for IVF following vitamin D supplementation rather than placebo (Fig. 2).

\section{Discussion}

The results of this trial demonstrated the beneficial effects of 50,000 IU vitamin D supplementation every other week for 8 weeks on improving insulin metabolism and some of the markers of lipid profile among infertile women diagnosed with PCOS who were candidate for IVF. These benefits might not be evident upon having sufficient vitamin D levels. To our best knowledge, this study has reported the effects of vitamin D supplementation on $\mathrm{AMH}$, glycemic control, lipid profiles and gene expression of insulin and lipid metabolism in infertile women for the first time.

Polycystic ovary syndrome predisposes patients to different metabolic abnormalities, such as insulin resistance and dyslipidemia [27, 28]. This study demonstrated that 50,000 IU vitamin D supplementation every other week for 8 weeks resulted in significant decreases in serum AMH, insulin levels and HOMA-IR score, and a significant increase in QUICKI in infertile women with PCOS who were

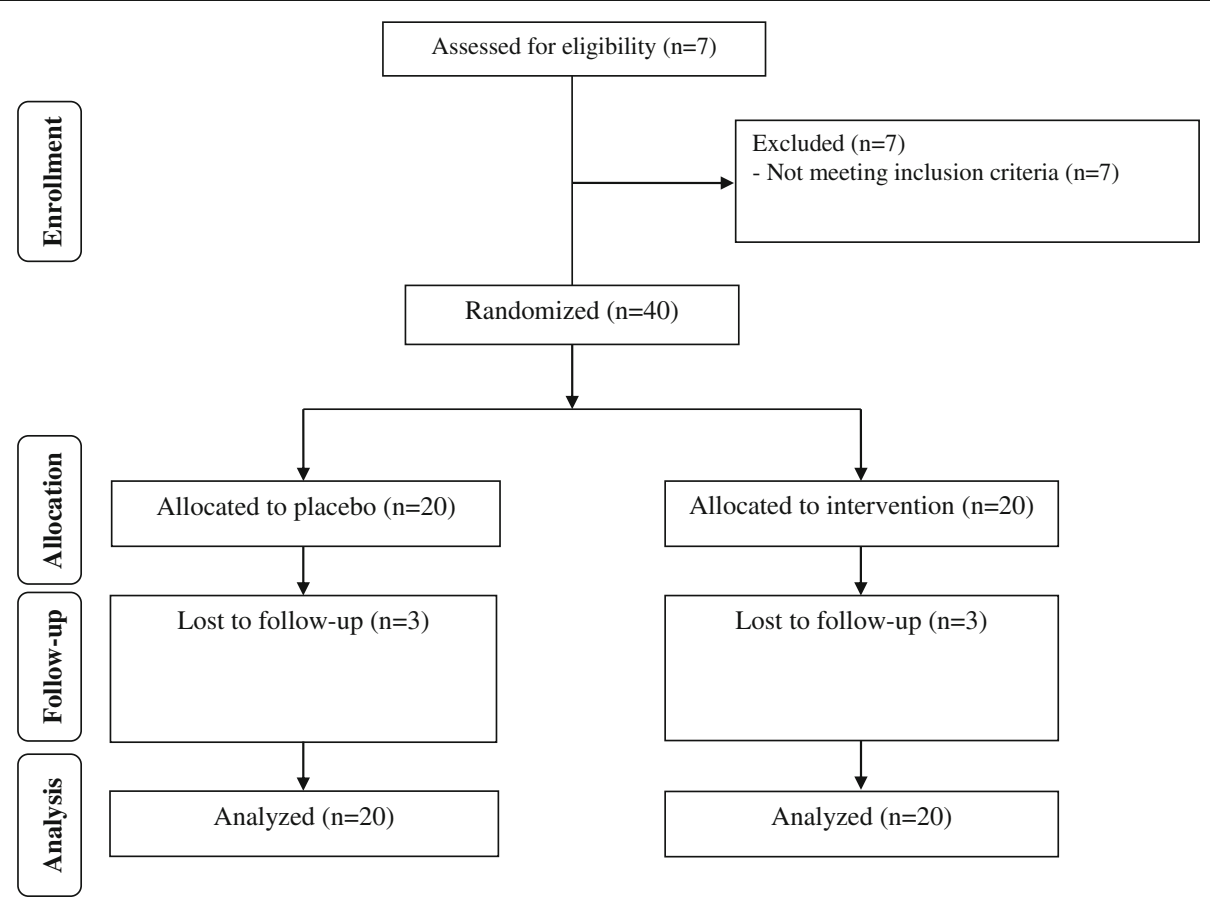

Fig. 1 Summary of patient flow diagram 
Table 2 General characteristics of study participants

\begin{tabular}{llll}
\hline & Placebo group $(\mathrm{n}=20)$ & Vitamin D group $(\mathrm{n}=20)$ & $P^{a}$ \\
\hline Age $(\mathrm{y})$ & $30.1 \pm 3.4$ & $29.9 \pm 4.4$ & 0.87 \\
Height $(\mathrm{cm})$ & $159.2 \pm 2.0$ & $160.4 \pm 3.3$ & 0.16 \\
Weight at study baseline $(\mathrm{kg})$ & $72.0 \pm 6.9$ & $71.3 \pm 9.9$ & 0.77 \\
Weight at end-of-trial $(\mathrm{kg})$ & $72.2 \pm 6.8$ & $71.5 \pm 9.6$ & 0.79 \\
Weight change $(\mathrm{kg})$ & $0.1 \pm 0.8$ & $0.2 \pm 0.9$ & 0.70 \\
BMl at study baseline $\left(\mathrm{kg} / \mathrm{m}^{2}\right)$ & $28.4 \pm 2.6$ & $27.7 \pm 3.9$ & 0.49 \\
BMl at end-of-trial $\left(\mathrm{kg} / \mathrm{m}^{2}\right)$ & $28.5 \pm 2.6$ & $27.8 \pm 3.7$ & 0.50 \\
BMl change $\left(\mathrm{kg} / \mathrm{m}^{2}\right)$ & $0.1 \pm 0.3$ & $0.1 \pm 0.3$ & 0.68 \\
\hline
\end{tabular}

Data are means \pm SDs

${ }^{a}$ Obtained from independent $t$-test

candidate for IVF. Further, vitamin D supplementation significantly increased gene expression levels of PPAR- $\gamma$ and GLUT-1. In the agreement with our study, Gupta et al. [29] demonstrated that vitamin $\mathrm{D}$ supplementation at a dosage of 60,000 IU weekly for 12 weeks significantly reduced insulin levels and HOMA-IR, and significantly increased QUICKI in vitamin D-deficient women with PCOS. In another study, taking vitamin D supplements at a dosage of 20,000 IU per week for 24 weeks by women with PCOS, having serum calcium levels $<2.65 \mathrm{mmol} / \mathrm{L}$ and uncertain serum $25(\mathrm{OH}) \mathrm{D}$ status at baseline, improved glucose metabolism and menstrual frequency [30]. However, there are also studies with discrepant findings. Garg et al. [31], showed that vitamin $\mathrm{D}$ supplementation at a dose of $4000 \mathrm{IU} /$ day for 6 months to vitamin D-deficient women with PCOS did not have any significant effect on insulin resistance and insulin secretion. Insulin resistance is associated with increased synthesis of vasoconstriction factors, which might be the reason for vascular stiffness in women diagnosed with PCOS [32]. Therefore, improved insulin metabolism by vitamin $\mathrm{D}$ supplementation may decrease the risk of metabolic complications subsequent to insulin resistance in these patients. The mechanisms involved in the impact of vitamin $\mathrm{D}$ on insulin metabolism could be direct and indirect effects of vitamin D on stimulating insulin release through improved vitamin $\mathrm{D}$ receptor expression, enhancing insulin sensitivity for glucose transportation and suppressing the release of pro-inflammatory cytokines [33].

We found that 50,000 IU vitamin D supplementation every other week for 8 weeks significantly decreased totaland LDL-cholesterol levels in infertile women with PCOS who were candidate for IVF, compared with the placebo, though other lipid profiles parameters remained unchanged. Moreover, vitamin D supplementation significantly increased gene expression levels of LDLR. Similar to our findings, vitamin D supplementation at a dosage of $4000 \mathrm{IU} /$ day for 12 weeks significantly decreased total cholesterol

Table 3 Glycemic control, markers of cardio-metabolic risk and oxidative stress at baseline and after the 8-week intervention in infertile polycystic ovary syndrome women candidate for in vitro fertilization that received either vitamin D supplements or placebo

\begin{tabular}{|c|c|c|c|c|c|c|c|}
\hline & Placebo grou & $=20)$ & & Vitamin D gro & $(n=20)$ & & $P^{a}$ \\
\hline & Baseline & End-of-trial & Change & Baseline & End-of-trial & Change & \\
\hline Vitamin D (ng/mL) & $11.0 \pm 2.4$ & $10.9 \pm 2.1$ & $-0.1 \pm 0.6$ & $10.5 \pm 2.5$ & $21.7 \pm 5.9$ & $11.2 \pm 5.0$ & $<0.001$ \\
\hline $\mathrm{AMH}(\mathrm{ng} / \mathrm{mL})$ & $8.7 \pm 2.7$ & $8.6 \pm 2.5$ & $-0.1 \pm 0.5$ & $7.7 \pm 3.4$ & $7.0 \pm 3.1$ & $-0.7 \pm 1.2$ & 0.02 \\
\hline FPG (mg/dL) & $92.9 \pm 5.5$ & $93.5 \pm 5.6$ & $0.5 \pm 3.0$ & $90.3 \pm 10.5$ & $89.4 \pm 10.6$ & $-0.9 \pm 7.4$ & 0.42 \\
\hline Insulin ( $\mu \mid \mathrm{U} / \mathrm{mL})$ & $11.4 \pm 1.9$ & $11.1 \pm 2.0$ & $-0.3 \pm 0.9$ & $11.2 \pm 2.2$ & $9.8 \pm 2.7$ & $-1.4 \pm 1.6$ & 0.007 \\
\hline HOMA-IR & $2.6 \pm 0.5$ & $2.5 \pm 0.4$ & $-0.1 \pm 0.2$ & $2.5 \pm 0.7$ & $2.2 \pm 0.7$ & $-0.3 \pm 0.3$ & 0.008 \\
\hline QUICKI & $0.33 \pm 0.008$ & $0.33 \pm 0.009$ & $0.001 \pm 0.004$ & $0.33 \pm 0.01$ & $0.34 \pm 0.02$ & $0.009 \pm 0.01$ & 0.04 \\
\hline Triglycerides (mg/dL) & $111.5 \pm 35.5$ & $117.4 \pm 34.8$ & $5.9 \pm 13.2$ & $105.3 \pm 33.5$ & $107.5 \pm 38.1$ & $2.1 \pm 17.4$ & 0.44 \\
\hline VLDL-cholesterol (mg/dL) & $22.3 \pm 7.1$ & $23.5 \pm 6.9$ & $1.2 \pm 2.6$ & $21.1 \pm 6.7$ & $21.5 \pm 7.6$ & $0.4 \pm 3.5$ & 0.44 \\
\hline Total cholesterol (mg/dL) & $197.1 \pm 36.3$ & $200.0 \pm 36.5$ & $2.9 \pm 10.9$ & $203.6 \pm 26.6$ & $198.5 \pm 24.7$ & $-5.1 \pm 12.6$ & 0.03 \\
\hline LDL-cholesterol (mg/dL) & $124.9 \pm 35.9$ & $127.4 \pm 35.3$ & $2.5 \pm 10.6$ & $133.1 \pm 21.1$ & $128.7 \pm 20.8$ & $-4.5 \pm 10.3$ & 0.04 \\
\hline HDL-cholesterol (mg/dL) & $49.9 \pm 7.5$ & $49.1 \pm 8.2$ & $-0.8 \pm 3.9$ & $49.4 \pm 5.7$ & $48.4 \pm 5.4$ & $-1.0 \pm 2.8$ & 0.81 \\
\hline
\end{tabular}

Data are means \pm SDs

a Obtained from repeated measures ANOVA test

$A M H$ anti-Müllerian hormone, FPG fasting plasma glucose, HOMA-IR homeostasis model of assessment-estimated insulin resistance, QUICKI quantitative insulin sensitivity check index 


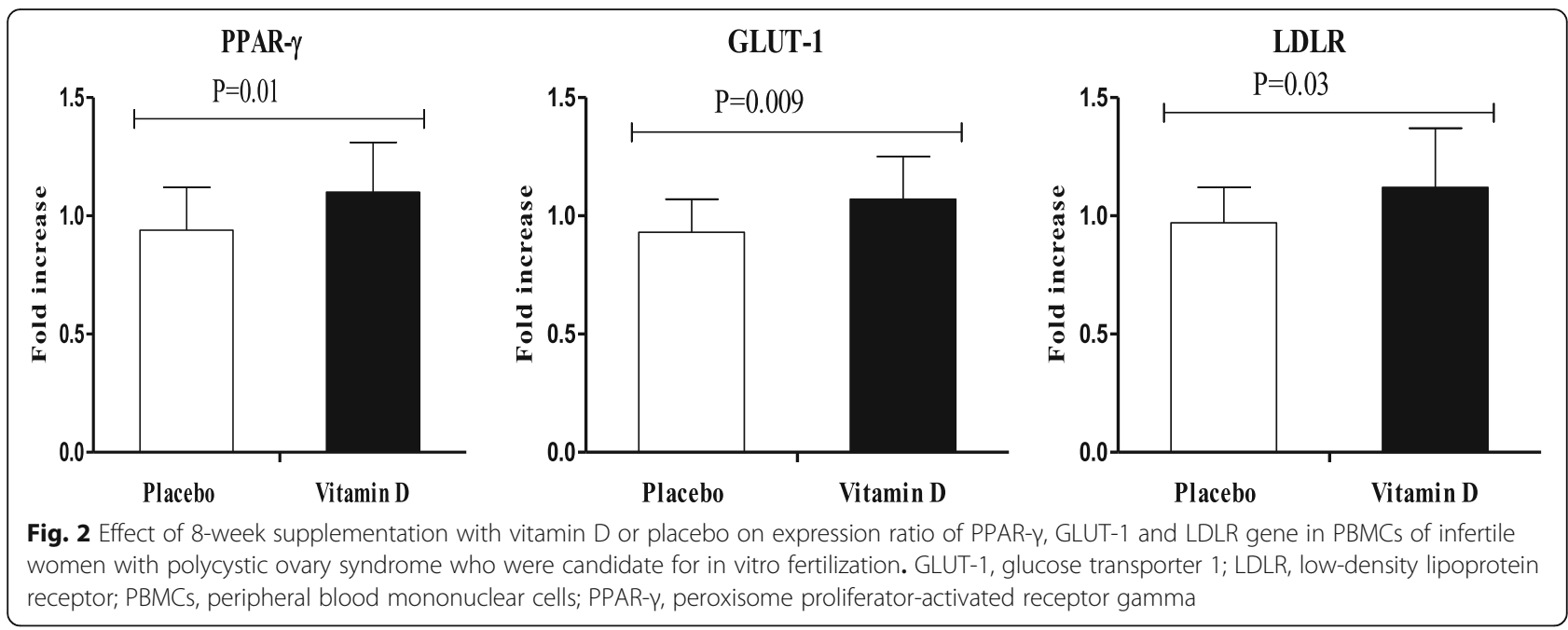

concentrations in vitamin $\mathrm{D}$ deficient $\mathrm{HIV}$-infected patients [34]. In a meta-analysis conducted by Akbari et al. [35], vitamin D supplementation significantly decreased LDL-cholesterol levels in gestational diabetes patients, though other lipid profiles parameters did not change. There are also contrary studies demonstrating that vitamin D supplementation (100,000 IU loading dose, followed by 20,000 IU/week) did not improve CVD risk factors including blood pressure, lipid profiles and glucose metabolism parameters in vitamin D insufficient population [36]. Current evidence suggested that increased insulin and androgens levels may negatively influence lipid profiles in women with PCOS [37]. Fatty acids byproducts have an important role in inflammation and reproduction [37]. Improved insulin sensitivity and decreased production of PTH by vitamin D might decrease total- and LDL-cholesterol [38]. Further, insulin reduces biosynthesis of cholesterol via increased 3-hydroxy-3-methylglutaryl CoA (HMG-CoA) reductase activity [39], which in turn decreases total- and LDL-cholesterol levels.

There are a few limitations in this study. First, the evaluation of insulin resistance in the current study, was only based on HOMA-IR. We did not assess any direct dynamic test, such as glucose tolerance test or hyperinsulinemic euglycemic clamp. Second, some of the insignificant results might be related to the short duration of intervention. So, further studies are required with longer duration and higher sample size to confirm our findings. In addition, all the participants were vitamin D deficient, so the observed effects might be correlated with the correction of vitamin D deficiency, rather than the supplementation per se. This should be considered in the interpretation of our finding.

\section{Conclusions}

Overall, the findings of this trial supported that 50,000 IU vitamin D supplementation every other week for 8 weeks had beneficial effects on insulin metabolism, and some parameters of lipid profiles among infertile women with PCOS who were candidate for IVF. These benefits might not be evident upon having sufficient vitamin D levels.

\section{Abbreviations}

$25(\mathrm{OH})$ D: 25-hydroxyvitamin D; AMH: anti-Müllerian hormone; FPG: fasting plasma glucose; GAPDH: glyceraldehyde-3-phosphate dehydrogenase; HOMA-IR: homeostasis model of assessment-estimated insulin resistance; HMG-COA: 3-hydroxy-3-methylglutaryl CoA; ITT: intention-to-treat; QUICKI: quantitative insulin sensitivity check index; GLUT-1: glucose transporter 1; LDLR: low-density lipoprotein receptor; PBMCs: peripheral blood mononuclear cells; PPAR-y: peroxisome proliferator-activated receptor gamma

\section{Acknowledgements}

Research reported in this publication was supported by Kashan University of Medical Sciences, Kashan, Iran.

\section{Funding}

This study was founded by a grant from the Vice-chancellor for Research, Kashan University of Medical Sciences, Kashan, Iran.

Availability of data and materials

The primary data for this study is available from the authors on direct request.

Authors' contributions

ZA and EA contributed in conception, design, statistical analysis and drafting of the manuscript. MD, NM, FF, SZ-M and MA-S contributed in data collection and manuscript drafting. All authors approved the final version for submission.

Ethics approval and consent to participate

This study was considered exempt by the KAUMS Institutional Review Board.

Consent for publication

Not applicable.

Competing interests

The authors declare that they have no competing interests.

\section{Publisher's Note}

Springer Nature remains neutral with regard to jurisdictional claims in published maps and institutional affiliations. 


\section{Author details}

'Research Center for Biochemistry and Nutrition in Metabolic Diseases, Kashan University of Medical Sciences, Kashan, I.R, Iran. ${ }^{2}$ School of Public Health, University of Saskatchewan, Saskatoon, SK, Canada. ${ }^{3}$ Department of Gynecology and Obstetrics, School of Medicine, Kashan University of Medical Sciences, Kashan, I.R, Iran. ${ }^{4}$ Laser Application in Medical Science Research Center, Shahid Beheshti University of Medical Sciences, Tehran, Iran. ${ }^{5}$ Taleghani Educational Hospital, IVF Center, Shahid Beheshti University of Medical Sciences, Tehran, Iran.

\section{Received: 23 July 2018 Accepted: 27 September 2018} Published online: 04 October 2018

\section{References}

1. Sheehan MT. Polycystic ovarian syndrome: diagnosis and management. Clin Med Res. 2004;2:13-27

2. Fica S, Albu A, Constantin M, Dobri GA. Insulin resistance and fertility in polycystic ovary syndrome. J Med Life. 2008;1:415-22.

3. Ceelen M, van Weissenbruch MM, Roos JC, Vermeiden JP, van Leeuwen FE. Delemarre-van de Waal HA. Body composition in children and adolescents born after in vitro fertilization or spontaneous conception. J Clin Endocrinol Metab. 2007:92:3417-23.

4. Ceelen M, van Weissenbruch MM, Vermeiden JP, van Leeuwen FE. Delemarre-van de Waal HA. Cardiometabolic differences in children born after in vitro fertilization: follow-up study. J Clin Endocrinol Metab. 2008;93: 1682-8.

5. Rexhaj E, Paoloni-Giacobino A, Rimoldi SF, Fuster DG, Anderegg M, Somm E, et al. Mice generated by in vitro fertilization exhibit vascular dysfunction and shortened life span. J Clin Invest. 2013;123:5052-60.

6. Scott KA, Yamazaki Y, Yamamoto M, Lin Y, Melhorn SJ, Krause EG, et al. Glucose parameters are altered in mouse offspring produced by assisted reproductive technologies and somatic cell nuclear transfer. Biol Reprod. 2010;83:220-7

7. Vlaisavljevic $V$, Kovac $V$, Sajko MC. Impact of insulin resistance on the developmental potential of immature oocytes retrieved from human chorionic gonadotropin-primed women with polycystic ovary syndrome undergoing in vitro maturation. Fertil Steril. 2009:91:957-9.

8. Halloran BP, DeLuca HF. Effect of vitamin D deficiency on fertility and reproductive capacity in the female rat. J Nutr. 1980;110:1573-80.

9. Daftary GS, Taylor HS. Endocrine regulation of HOX genes. Endocr Rev. 2006 27:331-55.

10. Kinuta K, Tanaka H, Moriwake T, Aya K, Kato S, Seino Y. Vitamin D is an important factor in estrogen biosynthesis of both female and male gonads. Endocrinology. 2000;141:1317-24

11. Johnson LE, DeLuca HF. Vitamin D receptor null mutant mice fed high levels of calcium are fertile. J Nutr. 2001:131:1787-91.

12. Wehr E, Pilz S, Schweighofer N, Giuliani A, Kopera D, Pieber TR, et al. Association of hypovitaminosis D with metabolic disturbances in polycystic ovary syndrome. Eur J Endocrinol. 2009;161:575-82.

13. Yildizhan $R$, Kurdoglu M, Adali E, Kolusari A, Yildizhan B, Sahin HG, et al. Serum 25-hydroxyvitamin D concentrations in obese and non-obese women with polycystic ovary syndrome. Arch Gynecol Obstet. 2009;280:559-63.

14. Turer CB, Lin H, Flores G. Prevalence of vitamin D deficiency among overweight and obese US children. Pediatrics. 2013;131:e152-61.

15. Zadeh-Vakili A, Ramezani Tehrani F, Daneshpour MS, Zarkesh M, Saadat N, Azizi F. Genetic polymorphism of vitamin D receptor gene affects the phenotype of PCOS. Gene. 2013;515:193-6.

16. Aleyasin A, Hosseini MA, Mahdavi A, Safdarian L, Fallahi P, Mohajeri MR, et al. Predictive value of the level of vitamin $D$ in follicular fluid on the outcome of assisted reproductive technology. Eur J Obstet Gynecol Reprod Biol. 2011;159:132-7.

17. Anifandis GM, Dafopoulos K, Messini Cl, Chalvatzas N, Liakos N, Pournaras S, et al. Prognostic value of follicular fluid $25-\mathrm{OH}$ vitamin D and glucose levels in the IVF outcome. Reprod Biol Endocrinol. 2010;8:91.

18. Butts SF, Seifer DB, Koelper N, Senapati S, Sammel MD, Hoofnagle AN, et al. Vitamin D deficiency is associated with poor ovarian stimulation outcome in pcos but not unexplained infertility. J Clin Endocrinol Metab. 2018. https://doi.org/10.1210/jc.2018-00750.

19. He C, Lin Z, Robb SW, Ezeamama AE. Serum vitamin D levels and polycystic ovary syndrome: a systematic review and meta-analysis. Nutrients. 2015;7: 4555-77.
20. Seyyed Abootorabi M, Ayremlou P, Behroozi-Lak T, Nourisaeidlou S. The effect of vitamin D supplementation on insulin resistance, visceral fat and adiponectin in vitamin D deficient women with polycystic ovary syndrome: a randomized placebo-controlled trial. Gynecol Endocrinol. 2018:34:489-94.

21. Xue $Y, X u$ P, Xue K, Duan $X, C a o ~ J, ~ L u a n ~ T$, et al. Effect of vitamin D on biochemical parameters in polycystic ovary syndrome women: a metaanalysis. Arch Gynecol Obstet. 2017;295:487-96.

22. Taylor PN, Davies JS. A review of the growing risk of vitamin D toxicity from inappropriate practice. Br J Clin Pharmacol. 2018;84:1121-7.

23. Rotterdam ESHRE/ASRM-Sponsored PCOS Consensus Workshop Group. Revised 2003 consensus on diagnostic criteria and long-term health risks related to polycystic ovary syndrome. Fertil Steril. 2004;81:19-25.

24. Pisprasert V, Ingram KH, Lopez-Davila MF, Munoz AJ, Garvey WT. Limitations in the use of indices using glucose and insulin levels to predict insulin sensitivity: impact of race and gender and superiority of the indices derived from oral glucose tolerance test in African Americans. Diabetes Care. 2013; 36:845-53.

25. Dunkley PR, Jarvie PE, Robinson PJ. A rapid Percoll gradient procedure for preparation of synaptosomes. Nat Protoc. 2008;3:1718-28.

26. Maktabi M, Chamani M, Asemi Z. The effects of vitamin D supplementation on metabolic status of patients with polycystic ovary syndrome: a randomized, double-blind, placebo-controlled trial. Horm Metab Res. 2017; 49:493-8.

27. Asemi Z, Foroozanfard F, Hashemi T, Bahmani F, Jamilian M, Esmaillzadeh A Calcium plus vitamin D supplementation affects glucose metabolism and lipid concentrations in overweight and obese vitamin D deficient women with polycystic ovary syndrome. Clin Nutr. 2015;34:586-92.

28. Foroozanfard F, Jamilian M, Bahmani F, Talaee $R$, Talaee $N$, Hashemi $T$, et al. Calcium plus vitamin D supplementation influences biomarkers of inflammation and oxidative stress in overweight and vitamin D-deficient women with polycystic ovary syndrome: a randomized double-blind placebo-controlled clinical trial. Clin Endocrinol. 2015;83:888-94.

29. Gupta T, Rawat M, Gupta N, Arora S. Study of effect of vitamin D supplementation on the clinical, hormonal and metabolic profile of the PCOS women. J Obstet Gynaecol India. 2017;67:349-55.

30. Wehr E, Pieber TR, Obermayer-Pietsch B. Effect of vitamin D3 treatment on glucose metabolism and menstrual frequency in polycystic ovary syndrome women: a pilot study. J Endocrinol Investig. 2011;34:757-63.

31. Garg G, Kachhawa G, Ramot R, Khadgawat R, Tandon N, Sreenivas V, et al. Effect of vitamin D supplementation on insulin kinetics and cardiovascular risk factors in polycystic ovarian syndrome: a pilot study. Endocr Connect. 2015:4:108-16

32. Kelly CJ, Speirs A, Gould GW, Petrie JR, Lyall H, Connell JM. Altered vascular function in young women with polycystic ovary syndrome. J Clin Endocrinol Metab. 2002;87:742-6

33. Alvarez JA, Ashraf A. Role of vitamin d in insulin secretion and insulin sensitivity for glucose homeostasis. Int J Endocrinol. 2010;2010:351385

34. Longenecker CT, Hileman CO, Carman TL, Ross AC, Seydafkan S, Brown TT, et al. Vitamin D supplementation and endothelial function in vitamin D deficient HIV-infected patients: a randomized placebo-controlled trial. Antivir Ther. 2012;17:613-21.

35. Akbari M, Mosazadeh M, Lankarani KB, Tabrizi R, Samimi M, Karamali M, et al. The effects of vitamin D supplementation on glucose metabolism and lipid profiles in patients with gestational diabetes: a systematic review and metaanalysis of randomized controlled trials. Horm Metab Res. 2017:49:647-53.

36. Kubiak JM, Thorsby PM, Kamycheva E, Jorde R. Vitamin D supplementation does not improve CVD risk factors in vitamin D insufficient subjects. Endocr Connect. 2018;7:840-9.

37. Li S, Chu Q, Ma J, Sun Y, Tao T, Huang R, et al. Discovery of novel lipid profiles in PCOS: do insulin and androgen oppositely regulate bioactive lipid production? J Clin Endocrinol Metab. 2017;102:810-21.

38. Wang $\mathrm{H}$, Xia N, Yang Y, Peng DQ. Influence of vitamin D supplementation on plasma lipid profiles: a meta-analysis of randomized controlled trials. Lipids Health Dis. 2012;11:42. https://doi.org/10.1186/1476-511X-11-42.

39. Kaplan M, Kerry R, Aviram M, Hayek T. High glucose concentration increases macrophage cholesterol biosynthesis in diabetes through activation of the sterol regulatory element binding. Protein 1 (SREBP 1): inhibitory effect of insulin J Cardiovasc Pharmacol. 2008:52:324-32. 